\title{
Thermal rates for baryon and anti-baryon production
}

\author{
Joseph Kapusta and Igor Shovkovy* \\ School of Physics and Astronomy, University of Minnesota \\ Minneapolis, Minnesota 55455, USA
}

\begin{abstract}
We use a form of the fluctuation-dissipation theorem to derive formulas giving the rate of production of spin-1/2 baryons in terms of the fluctuations of either meson or quark fields. The most general formulas do not assume thermal or chemical equilibrium. When evaluated in a thermal ensemble we find equilibration times on the order of $10 \mathrm{fm} / \mathrm{c}$ near the critical temperature in QCD.
\end{abstract}

PACS: 12.38.Mh, 25.75.Dw, 24.85.+p

Typeset using REVTEX 


\section{INTRODUCTION}

Stable or long-lived baryons are readily detectable in high energy nuclear collisions, such as those at the CERN SPS (Super Proton Synchrotron) and at the Brookhaven National Laboratory RHIC (Relativistic Heavy Ion Collider). They have masses on the order of 1 $\mathrm{GeV}$, which makes them relatively sensitive to the temperatures and expansion rates in such collisions. Recent measurements with $\mathrm{Au}+\mathrm{Au}$ collisions at $\sqrt{s}=200 \mathrm{~A} \mathrm{GeV}$ at $\mathrm{RHIC}$ are consistent with protons, lambdas, sigmas, cascades, and omegas, and their anti-particles, all being in chemical equilibrium at a temperature of $170 \pm 10 \mathrm{MeV}$ [1]. This temperature is close to the expected critical or rapid crossover temperature in QCD between a quark-gluon phase and a hadronic phase. How is it possible to understand such near perfect equilibration on the relatively short time scales of high energy nuclear collisions?

One approach is kinetic theory, which was reviewed in some detail already by Koch, Müller and Rafelski [2]. At finite temperatures, but still in the hadronic phase, there exist many mesons, especially $\pi, K, \rho$ and $\omega$. Two body reactions like $\pi \pi \rightarrow B \bar{B}$ dominate at moderate temperatures, say $100 \mathrm{MeV}$ and less. As the temperature goes up so does the density of mesons. Multi-particle reactions, such as $\pi \pi \pi K \bar{K} \rightarrow B \bar{B}$ become increasingly important. These multi-particle reactions cannot be measured directly in the laboratory, of course, but they can be related to the inverse reactions by detailed balance. Some of these inverse reactions, those involving proton plus anti-proton annihilation into mesons, were measured at LEAR (Low Energy Anti-proton Ring) at CERN. Rapp and Shuryak [3] have estimated that the sum of all reactions of the type $n \pi \rightarrow p \bar{p}$ are able to yield fast equilibration times for anti-protons at temperatures of order 150 to $170 \mathrm{MeV}$, perhaps as short as several $\mathrm{fm} / \mathrm{c}$. However, there are at least three difficulties with applying kinetic theory, using vacuum reactions rates, to high energy density matter. First, experimental data on the annihilation of hyperons into mesons is practically nonexistent. One must rely on approximate $\mathrm{SU}(3)$ flavor symmetry to estimate the rates based on nucleon data. Second, there is no sound practical method to implement microscopic reactions involving 
initial states with more than two particles in a microscopic cascade computer code. Two body initial state reactions are assumed to occur when the colliding particles are within a distance $\sqrt{\sigma / \pi}$ of each other, where $\sigma$ is the cross section. For three or more particles the criterion becomes ambiguous. Third, and perhaps most importantly, the very concept of localized interactions occurring as in vacuum is no longer applicable. When particle densities reach 1 per cubic fermi with particles whose physical extent is also about $1 \mathrm{fm}$, it is not possible to define in and out states as in the vacuum. The interaction radius is comparable to the physical matter radius of the hadrons. The best that kinetic theory can do for hadrons at high temperatures is to tell whether the equilibration time is small or large but probably cannot give a quantitative number.

Another approach is with DCC (disoriented chiral condensates) [4]. In this approach baryons are described as topological defects of the chiral field using the model of Skyrme. Domains are formed at some early time, and as the matter expands all the domains must eventually line up with the surrounding vacuum. During this process defects are formed with a probability that was first calculated in the context of the early universe [5]. The probability of defect production is inversely proportional to the cube of the domain size. Smaller domains yield more baryons and anti-baryons. Together with Wong, one of us showed that the anomalously large number of $\Omega$ and $\bar{\Omega}$ observed at the SPS could be understood in terms of this mechanism [6]. The typical domain size needed is $2 \mathrm{fm}$, which is just in the range predicted by many different approaches to DCC formation [0]. However, it is difficult to make much more quantitative calculations in this approach without very extensive numerical simulations. Even then, one may question whether a low energy effective meson field theory can reasonably describe variations over length scales as small as $2 \mathrm{fm}$. Plus the relevant frequencies involved are twice the proton mass.

At first it would seem that these two approaches are almost orthogonal to each other. In fact they are trying to describe the same physics, baryon production at high energy density, starting from two opposite perspectives. Kinetic theory attempts to describe the process with many mesons that propagate freely between localized collisions. The DCC approach 
assumes that the meson density is so high that they may be treated collectively as a classical field; baryons arise as topological defects of this field.

In this paper we attempt a more general description than either of the above. We use a version of the fluctuation-dissipation theorem, basically the same formalism as used to compute the production rate of real and virtual photons in hot matter. The baryon production rate can be expressed in terms of the fluctuations in the local meson fields or in terms of fluctuations in the local quark fields. The formulas derived can be applied to systems in equilibrium or out of equilibrium. In the former case we evaluate the rates numerically; this is possible because of the Boltzmann weighting of states. In the latter case one must specify the probability of different states according to the preparation of the non-equilibrium system under consideration. We first compute the rates for non-strange baryons only, then we generalize to hyperons using SU(3) flavor symmetry in the interaction Lagrangian.

\section{NUCLEON PRODUCTION}

In this section we analyze the production of nucleons and anti-nucleons by vector and axial-vector fields or currents. We first derive general formulas that express the production rate in terms of fluctuations of these fields or currents. Formulas are given for arbitrary weighting of states. The formulas are then evaluated in thermal equilibrium with a Boltzmann weighting of states. The vector and axial-vector fields or currents are first expressed explicitly in terms of pion fields using the nonlinear sigma model. They are alternately expressed in terms of the quark fields using vector meson dominance to determine the relevant couplings.

\section{A. General formulas}

With a view toward the conventions used in the nonlinear sigma model we write the coupling of nucleons to vector $V_{\mu}^{a}$ and axial-vector $A_{\mu}^{a}$ currents or fields as 


$$
\mathcal{L}=-\bar{\psi} \gamma^{\mu} \frac{\tau^{a}}{2} \psi V_{\mu}^{a}+g_{A} \bar{\psi} \gamma^{\mu} \gamma^{5} \frac{\tau^{a}}{2} \psi A_{\mu}^{a}
$$

where $g_{A} \approx 1.26$ is the axial coupling constant relative to the vector. We do not need to specify anything more about $V_{\mu}^{a}$ and $A_{\mu}^{a}$. They are given fields or currents that produce baryons via the above coupling. The production rate of nucleon/anti-nucleon pairs is calculated in the same manner as dileptons [8] using linear response theory, equivalently a version of the fluctuation-dissipation theorem. We show explicitly the steps for production in the axial-vector channel.

The matrix element for the process $i \rightarrow f+$ baryon + anti-baryon is

$$
S_{f i}=g_{A}\left\langle f\left|\int d^{4} x A_{\mu}^{a}(x) J_{a}^{5 \mu}(x)\right| i\right\rangle
$$

Here $J_{a}^{5 \mu}$ is an abbreviation for the axial baryonic current as expressed in the Lagrangian above. The initial and final states are arbitrary, usually fully interacting states except for the specific interaction in Eq. (1). The axial baryonic current, corresponding to the baryons in the final state, is

$$
J_{a}^{5 \mu}(x)=\frac{1}{V} \sqrt{\frac{m_{N}^{2}}{E_{1} E_{2}}} e^{-i x \cdot\left(p_{1}+p_{2}\right)} \bar{u}\left(p_{1}, s_{1}\right) \gamma^{5} \gamma^{\mu} \frac{\tau_{a}}{2} v\left(p_{2}, s_{2}\right) .
$$

As a consequence of the translational invariance we can write

$$
\left\langle f\left|A_{\mu}^{a}(x)\right| i\right\rangle=e^{i x \cdot k}\left\langle f\left|A_{\mu}^{a}(0)\right| i\right\rangle .
$$

Here $k \equiv k_{i}-k_{f}$ is the difference of the four-momenta of the initial and final states. The transition rate is

$$
R_{f i}=\frac{\left|S_{f i}\right|^{2}}{T V}
$$

where $T$ is the time interval and $V$ is the volume. Explicitly

$$
\begin{aligned}
R_{f i} & =g_{A}^{2} \int \frac{d^{4} x d^{4} x^{\prime}}{T V}\left\langle f\left|A_{\mu}^{a}\left(x+x^{\prime}\right) J_{a}^{5 \mu}\left(x+x^{\prime}\right)\right| i\right\rangle\left\langle i\left|A_{\nu}^{b}\left(x^{\prime}\right) J_{b}^{5 \nu}\left(x^{\prime}\right)\right| f\right\rangle \\
& =\frac{g_{A}^{2}}{V^{2}} \frac{m_{N}^{2}}{E_{1} E_{2}} \int d^{4} x e^{i x \cdot\left(k-p_{1}-p_{2}\right)}\left\langle f\left|A_{\mu}^{a}(0)\right| i\right\rangle\left\langle i\left|A_{\nu}^{b}(0)\right| f\right\rangle \\
& \times \bar{u}\left(p_{1}, s_{1}\right) \gamma^{5} \gamma^{\mu} \frac{\tau_{a}}{2} v\left(p_{2}, s_{2}\right) \bar{v}\left(p_{2}, s_{2}\right) \gamma^{5} \gamma^{\nu} \frac{\tau_{b}}{2} u\left(p_{1}, s_{1}\right)
\end{aligned}
$$


Averaging over initial states with an arbitrary weight $w_{i}$ and summing over final states, we arrive at the following differential rate.

$$
\begin{aligned}
d R & =\frac{g_{A}^{2} m_{N}^{2}}{E_{1} E_{2}} \frac{d^{3} p_{1} d^{3} p_{2}}{(2 \pi)^{6}} \sum_{i} w_{i} \sum_{f}(2 \pi)^{4} \delta^{4}\left(p_{1}+p_{2}-k\right)\left\langle f\left|A_{\mu}^{a}(0)\right| i\right\rangle \\
& \times\left\langle i\left|A_{\nu}^{b}(0)\right| f\right\rangle \operatorname{Tr}\left(\frac{\not p_{1}+m_{N}}{2 m_{N}} \gamma^{5} \gamma^{\mu} \frac{\tau_{a}}{2} \frac{\not p_{2}-m_{N}}{2 m_{N}} \gamma^{5} \gamma^{\nu} \frac{\tau_{b}}{2}\right)
\end{aligned}
$$

By introducing the correlation function

$$
A_{\mu \nu}^{(-) a b}(p)=-\sum_{i} w_{i} \sum_{f}(2 \pi)^{4} \delta^{4}(p-k)\left\langle f\left|A_{\mu}^{a}(0)\right| i\right\rangle\left\langle i\left|A_{\nu}^{b}(0)\right| f\right\rangle
$$

and evaluating the trace

$$
\begin{aligned}
& \operatorname{Tr}\left(\frac{\not p_{1}+m_{N}}{2 m_{N}} \gamma^{5} \gamma^{\mu} \frac{\tau_{a}}{2} \frac{\not p_{2}-m_{N}}{2 m_{N}} \gamma^{5} \gamma^{\nu} \frac{\tau_{b}}{2}\right) \\
= & \frac{\delta^{a b}}{2 m_{N}^{2}}\left[p_{1}^{\mu} p_{2}^{\nu}+p_{1}^{\nu} p_{2}^{\mu}+g^{\mu \nu}\left(m_{N}^{2}-p_{1} \cdot p_{2}\right)\right],
\end{aligned}
$$

we arrive at

$$
E_{1} E_{2} \frac{d R_{A}}{d^{3} p_{1} d^{3} p_{2}}=-\frac{g_{A}^{2}}{2(2 \pi)^{6}} A_{\mu \nu}^{(-) a a}\left(p_{1}+p_{2}\right)\left[p_{1}^{\mu} p_{2}^{\nu}+p_{1}^{\nu} p_{2}^{\mu}-g^{\mu \nu}\left(p_{1} \cdot p_{2}-m_{N}^{2}\right)\right]
$$

Apart from the rather trivial Lorentz tensor coming from the form of the axial baryonic current, all the physics is contained in the correlation function.

The corresponding rate arising from the vector interaction is easily obtained. The only differences are the replacement of the axial-vector with the axial field or current, $A \rightarrow V$, setting $g_{A}^{2}=1$, and changing the sign of the mass-squared term in the Lorentz tensor.

$$
E_{1} E_{2} \frac{d R_{V}}{d^{3} p_{1} d^{3} p_{2}}=-\frac{1}{2(2 \pi)^{6}} V_{\mu \nu}^{(-) a a}\left(p_{1}+p_{2}\right)\left[p_{1}^{\mu} p_{2}^{\nu}+p_{1}^{\nu} p_{2}^{\mu}-g^{\mu \nu}\left(p_{1} \cdot p_{2}+m_{N}^{2}\right)\right]
$$

These are the first significant results in this section. They are quite general, but they require knowledge of the correlation functions for the initial states, appropriately weighted, and final states for the specific system and conditions under consideration.

Now we evaluate the correlation functions in thermal equilibrium. The states $|i\rangle$ and $|f\rangle$ are conveniently assumed to be eigenstates of the Hamiltonian $\hat{H}$ with eigenvalues $E_{i}$ and $E_{f}$. The weight is 


$$
w_{i}=\frac{e^{-\beta E_{i}}}{Z}
$$

where $\beta$ is the inverse temperature and $Z$ is the partition function. Following standard practice, the retarded correlation function in position space is

$$
A_{\mu \nu}^{(R) a b}(x)=-i \frac{\theta\left(x^{0}\right)}{Z} \operatorname{Tr}\left(e^{-\beta \hat{H}}\left[A_{\mu}^{a}(x), A_{\nu}^{b}(0)\right]\right) .
$$

Its Fourier transform is

$$
\begin{aligned}
& A_{\mu \nu}^{(R) a b}(p)=-i \int_{0}^{\infty} d x^{0} d^{3} x e^{i\left[\left(p^{0}+i \varepsilon\right) x^{0}-\mathbf{x} \cdot \mathbf{p}\right]} \\
& \times \sum_{i, f}\left(w_{i}\left\langle i\left|A_{\mu}^{a}(x)\right| f\right\rangle\left\langle f\left|A_{\nu}^{b}(0)\right| i\right\rangle-w_{f}\left\langle i\left|A_{\mu}^{a}(x)\right| f\right\rangle\left\langle f\left|A_{\nu}^{b}(0)\right| i\right\rangle\right) \\
& =-i \int_{0}^{\infty} d x^{0} d^{3} x e^{i\left[\left(p^{0}-k^{0}+i \varepsilon\right) x^{0}-\mathbf{x} \cdot(\mathbf{p}-\mathbf{k})\right]} \sum_{i, f} w_{i}\left(1-e^{\beta k^{0}}\right) \\
& \times\left\langle i\left|A_{\mu}^{a}(0)\right| f\right\rangle\left\langle f\left|A_{\nu}^{b}(0)\right| i\right\rangle \\
& =\sum_{i, f} \frac{w_{i}\left(1-e^{\beta k^{0}}\right)(2 \pi)^{3} \delta^{3}(\mathbf{p}-\mathbf{k})}{p^{0}-k^{0}+i \varepsilon}\left\langle i\left|A_{\mu}^{a}(0)\right| f\right\rangle\left\langle f\left|A_{\nu}^{b}(0)\right| i\right\rangle .
\end{aligned}
$$

As before, $k=k_{i}-k_{f}$. The imaginary part is

$$
\operatorname{Im} A_{\mu \nu}^{(R) a b}(p)=-\frac{1}{2} \sum_{i, f} w_{i}\left(1-e^{\beta p^{0}}\right)(2 \pi)^{4} \delta^{4}(p-k)\left\langle i\left|A_{\mu}^{a}(0)\right| f\right\rangle\left\langle f\left|A_{\nu}^{b}(0)\right| i\right\rangle .
$$

Hence

$$
A_{\mu \nu}^{(-) a b}(p)=\frac{2}{1-e^{\beta p^{0}}} \operatorname{Im} A_{\mu \nu}^{(R) a b}(p) .
$$

Exactly the same relationship holds in the vector channel with the substitution of $A$ with $V$.

The sum of the rates of production by vector and axial-vector fields or currents can now be written as

$$
\begin{aligned}
& E_{1} E_{2} \frac{d R}{d^{3} p_{1} d^{3} p_{2}}=\frac{1}{(2 \pi)^{6}} \frac{1}{e^{\beta\left(E_{1}+E_{2}\right)}-1} \\
& \times\left\{\operatorname{Im} V_{\mu \nu}^{(R) a a}\left(p_{1}+p_{2}\right)\left[p_{1}^{\mu} p_{2}^{\nu}+p_{1}^{\nu} p_{2}^{\mu}-g^{\mu \nu}\left(p_{1} \cdot p_{2}+m_{N}^{2}\right)\right]\right. \\
& \left.+g_{A}^{2} \operatorname{Im} A_{\mu \nu}^{(R) a a}\left(p_{1}+p_{2}\right)\left[p_{1}^{\mu} p_{2}^{\nu}+p_{1}^{\nu} p_{2}^{\mu}-g^{\mu \nu}\left(p_{1} \cdot p_{2}-m_{N}^{2}\right)\right]\right\} .
\end{aligned}
$$

This is the second significant result in this section. In order to make further progress, we need specific knowledge of the vector and axial-vector fields or currents and their correlation functions. 


\section{B. Production in terms of pion fluctuations}

The self-interactions among pions in the nonlinear sigma model are contained in the Lagrangian

$$
\mathcal{L}=\frac{f_{\pi}^{2}}{4} \operatorname{Tr}\left(\partial_{\mu} U \partial^{\mu} U^{\dagger}+m_{\pi}^{2} U+m_{\pi}^{2} U^{\dagger}\right)
$$

where

$$
\begin{aligned}
U & =\xi^{2} \\
\xi & =\exp \left(\frac{i}{2 f_{\pi}} \pi^{a} \tau_{a}\right) .
\end{aligned}
$$

Here $\pi^{a}$ is the pion field and $f_{\pi} \approx 93 \mathrm{MeV}$ is the pion decay constant. The pions couple to the baryons as in Eq. (II) with the derived currents

$$
\begin{aligned}
A_{\mu}^{a} & \equiv \frac{i}{2} \operatorname{Tr}\left[\tau_{a}\left(\xi \partial_{\mu} \xi^{\dagger}-\xi^{\dagger} \partial_{\mu} \xi\right)\right]=\frac{1}{f_{\pi}} \partial_{\mu} \pi^{a}+\cdots, \\
V_{\mu}^{a} & \equiv-\frac{i}{2} \operatorname{Tr}\left[\tau_{a}\left(\xi \partial_{\mu} \xi^{\dagger}+\xi^{\dagger} \partial_{\mu} \xi\right)\right]=\frac{1}{f_{\pi}^{2}} \varepsilon^{a b c} \pi^{b} \partial_{\mu} \pi^{c}+\cdots .
\end{aligned}
$$

To first order in the pion field, this gives the usual derivative coupling on account of the Goldberger-Treiman relation $g_{A} m_{N}=f_{\pi} g_{\pi N N}$. By expanding the interaction in powers of the pion field one may derive the contribution to the production rate involving two, three, four, five, etc. number of pions in the initial state.

\section{Production in terms of quark fluctuations}

Since pions are made of quarks it should be possible to express the vector and axialvector fields or currents in terms of quark operators. We can make the connection using the

hypothesis of vector meson dominance (VMD). In the two-flavor world the currents can be expressed in terms of the $\rho, a_{1}$ and pion fields as

$$
\begin{aligned}
V_{\mu}^{a} & =-g_{\rho N N} \rho_{\mu}^{a}, \\
A_{\mu}^{a} & =\frac{g_{a_{1} N N}}{g_{A}} a_{1 \mu}^{a}+\frac{g_{\pi N N}}{g_{A} m_{N}} \partial_{\mu} \pi^{a} .
\end{aligned}
$$


Using the Goldberger-Trieman relation and VMD the quark currents are related to the hadronic fields.

$$
\begin{aligned}
\bar{q} \gamma_{\mu} \frac{\tau^{a}}{2} q & =\frac{m_{\rho}^{2}}{g_{\rho \pi \pi}} \rho_{\mu}^{a} \\
\bar{q} \gamma_{\mu} \gamma^{5} \frac{\tau^{a}}{2} q & =\frac{m_{a_{1}}^{2}}{g_{a_{1}}} a_{1 \mu}^{a}+\text { pion }
\end{aligned}
$$

Apart from the pion pole we obtain the desired relationship.

$$
\begin{aligned}
V_{\mu}^{a} & =-\frac{g_{\rho N N} g_{\rho \pi \pi}}{m_{\rho}^{2}} \bar{q} \gamma_{\mu} \frac{\tau^{a}}{2} q \\
A_{\mu}^{a} & =\frac{g_{a_{1} N N} g_{a_{1}}}{m_{a_{1}}^{2}} \bar{q} \gamma_{\mu} \gamma^{5} \frac{\tau^{a}}{2} q
\end{aligned}
$$

The imaginary part of the retarded correlator is given in terms of the spectral density $\rho(s)$ as

$$
\operatorname{Im} V_{\mu \nu}^{(R) a a}(k)=-3\left(g_{\mu \nu}-\frac{k_{\mu} k_{\nu}}{k^{2}}\right) \pi \rho_{V}\left(s=k^{2}\right),
$$

with a similar expression for the axial channel. The factor of 3 arises from the sum over isospin indices.

In our study of baryon/anti-baryon production, the spectral densities are needed only for $s>4 m_{N}^{2}$. Then $s$ is large enough that they may be computed using perturbative QCD. (The vector one may be measured directly in electron-positron collisions; in the region of $\sqrt{s}$ of several $\mathrm{GeV}$ a sum of hadronic resonances gives essentially the same answer, a nice manifestation of duality.) They are [9]

$$
\begin{aligned}
& \rho_{V}(s)=\left(\frac{g_{\rho N N} g_{\rho \pi \pi}}{m_{\rho}^{2}}\right)^{2} \frac{s}{8 \pi^{2}}\left(1+\frac{\alpha_{s}(s)}{\pi}+\cdots\right), \\
& \rho_{A}(s)=\left(\frac{g_{a_{1} N N} g_{a_{1}}}{g_{A} m_{a_{1}}^{2}}\right)^{2} \frac{s}{8 \pi^{2}}\left(1+\frac{\alpha_{s}(s)}{\pi}+\cdots\right) .
\end{aligned}
$$

Here $\alpha_{s}(s)$ is the QCD coupling evaluated at the scale $s$.

According to KSFR 10 $2 g_{\rho}^{2} f_{\pi}^{2}=m_{\rho}^{2}$. Furthermore it is usually assumed that $g_{\rho}$ is universal in the sense that $g_{\rho N N}=g_{\rho \pi \pi}$, a result that holds rather well numerically. Then the coefficient of the vector spectral density is especially simple. 


$$
\left(\frac{g_{\rho N N} g_{\rho \pi \pi}}{m_{\rho}^{2}}\right)^{2}=\frac{1}{4 f_{\pi}^{4}}
$$

In the absence of any better information it is quite reasonable to assume that the same holds in the axial-vector channel.

$$
\left(\frac{g_{a_{1} N N} g_{a_{1}}}{m_{a_{1}}^{2}}\right)^{2}=\frac{1}{4 f_{\pi}^{4}}
$$

This means that the vector and axial-vector currents have the same spectral density since the axial coupling $g_{A}$ then cancels out. Equal contributions is quite natural when the up and down quark masses are very small, as is the case in the real world.

The tensor algebra can now be done using $k=p_{1}+p_{2}$. It yields the third significant result of this section.

$$
E_{1} E_{2} \frac{d R}{d^{3} p_{1} d^{3} p_{2}}=\frac{3}{8(2 \pi)^{7}} \frac{1}{e^{\beta\left(E_{1}+E_{2}\right)}-1} \frac{s\left(s-m_{N}^{2}\right)}{f_{\pi}^{4}}\left(1+\frac{\alpha_{s}(s)}{\pi}+\cdots\right)
$$

This result is quite remarkable in that the rate is inversely proportional to the fourth power of $f_{\pi}$ and does not depend on any other hadronic parameters except the nucleon mass.

Finally, the overall rate of production may be computed by integrating over the momenta of the outgoing nucleons. Since the threshold energy, $2 m_{N}$, is much greater than the temperatures envisioned, $T<200 \mathrm{MeV}$, it suffices to drop the minus one in the Bose-Einstein distribution factor in the differential rate. Then, with $K_{n}$ denoting the Bessel function of the second kind, we get

$$
\begin{aligned}
R(\bar{N} N) & =\frac{9}{(2 \pi)^{5}}\left(1+\frac{\alpha_{s}\left(4 m_{N}^{2}\right)}{\pi}+\cdots\right) \frac{m_{N}^{4} T^{4}}{f_{\pi}^{4}}\left[\frac{m_{N}^{2}}{T^{2}} K_{1}^{2}\left(\frac{m_{N}}{T}\right)\right. \\
& \left.+4 \frac{m_{N}}{T} K_{1}\left(\frac{m_{N}}{T}\right) K_{2}\left(\frac{m_{N}}{T}\right)+\left(8+\frac{m_{N}^{2}}{T^{2}}\right) K_{2}^{2}\left(\frac{m_{N}}{T}\right)\right]
\end{aligned}
$$

In the nonrelativistic limit this becomes

$$
R(\bar{N} N)=\frac{9}{2(2 \pi)^{4}}\left(1+\frac{\alpha_{s}\left(4 m_{N}^{2}\right)}{\pi}+\cdots\right) \frac{m_{N}^{5} T^{3}}{f_{\pi}^{4}} \exp \left(-2 m_{N} / T\right) .
$$

This is the total rate for the production of $\bar{p} p, \bar{p} n, \bar{n} p$ and $\bar{n} n$. The individual rates are related as: $R(\bar{p} n)=R(\bar{n} p)=2 R(\bar{p} p)=2 R(\bar{n} n)$. 
It should be noted that we have evaluated $\alpha_{s}$ at threshold for nucleon/anti-nucleon production where the rate is a maximum. According to the latest analysis [11 $\alpha_{s}\left(m_{\tau}^{2}\right)=$ $0.35 \pm 0.03$. Since $m_{\tau}=1777 \mathrm{MeV}$ is very close to $2 m_{N}$ we use that as the numerical value in our later calculations. The first perturbative correction to the spectral density is only about $10 \%$.

It should also be noted that we have included the interactions involving the isospin $I=1$ currents only. If the isospin $I=0$ currents were included too one might expect the rates for $\bar{p} p$ and $\bar{n} n$ to increase while the rates for $\bar{p} n$ and $\bar{n} p$ to be unchanged. Indeed, this is what happens, and in fact the rates for $\bar{p} p$ and $\bar{p} n$ become approximately equal, but we defer the actual analysis to the next section.

Finally, we point out that since nucleons are composite objects they have form factors. These form factors will multiply the above rates and will serve to decrease them to some degree. We will defer the determination of the form factors to the next chapter. The reason is that there is accurate data on $\bar{p} p$ annihilations, and to do a precise analysis we must first include the isospin $I=0$ current.

\section{NUCLEON AND HYPERON PRODUCTION}

An amazing fact in heavy ion collisions at RHIC is that hyperons are produced in relatively great abundance. Measurements indicate that they are, for all practical purposes, in chemical equilibrium at a temperature of $170 \pm 10 \mathrm{MeV}$. Therefore it behooves for us to analyze hyperon production. In addition, we now will include coupling to the isospin $I=0$ vector and axial-vector currents or fields too. We side-stepped that contribution in the last section for clarity of presentation and to avoid making phenomenological estimates of the relevant couplings: assumption of $\mathrm{SU}(3)$ invariance of the interactions will help to resolve that issue. We will consider fluctuations in the $\mathrm{SU}(2)$ sector only as well as fluctuations in the full SU(3) sector. The results are rather different. 


\section{A. General SU(3) invariant couplings}

Flavor $\mathrm{SU}(3)$ is not nearly as good a symmetry as $\mathrm{SU}(2)$. However, experience over many decades of research has shown that it is usually a very good reproduction of experimental data to put all the flavor symmetry breaking in the mass terms but to insist that the interactions be flavor symmetric. That is what we shall do too.

The vector meson nonet is conventionally written as follows [12, 13].

$$
\mathcal{V}=\left(\begin{array}{ccc}
\frac{\rho^{0}}{\sqrt{2}}+\frac{\omega_{8}}{\sqrt{6}}+\frac{\omega_{s}}{\sqrt{3}} & \rho^{+} & K^{*+} \\
\rho^{-} & -\frac{\rho^{0}}{\sqrt{2}}+\frac{\omega_{8}}{\sqrt{6}}+\frac{\omega_{s}}{\sqrt{3}} & K^{* 0} \\
K^{*-} & \bar{K}^{* 0} & \frac{-2 \omega_{8}}{\sqrt{6}}+\frac{\omega_{s}}{\sqrt{3}}
\end{array}\right)
$$

The singlet and octet components are actually a mixture of the physical $\omega$ and $\phi$ mesons.

$$
\begin{aligned}
& \omega_{8}=\phi \cos \theta_{V}+\omega \sin \theta_{V} \\
& \omega_{s}=\omega \cos \theta_{V}-\phi \sin \theta_{V}
\end{aligned}
$$

Ideal mixing occurs when the $\omega$ has no $s \bar{s}$ component while the $\phi$ is pure $s \bar{s}$. The ideal mixing angle is $\tan \theta_{\text {ideal }}=1 / \sqrt{2}$, or $\theta_{\text {ideal }} \approx 35.3^{\circ}$. Experimentally the mixing angle seems to be about $39^{\circ}$ [11]. We will approximate the mixing as ideal to simplify formulas. Such

fine details are not likely to be important in the context we have in mind, namely, heavy ion collisions. Therefore we use the nonet representation.

$$
\mathcal{V}=\left(\begin{array}{ccc}
\frac{\rho^{0}}{\sqrt{2}}+\frac{\omega}{\sqrt{2}} & \rho^{+} & K^{*+} \\
\rho^{-} & -\frac{\rho^{0}}{\sqrt{2}}+\frac{\omega}{\sqrt{2}} & K^{* 0} \\
K^{*-} & \bar{K}^{* 0} & -\phi
\end{array}\right)
$$

A similar assumption of ideal mixing in the axial-vector meson nonet yields the following representation.

$$
\mathcal{A}=\left(\begin{array}{ccc}
\frac{a_{1}^{0}}{\sqrt{2}}+\frac{f_{1}(1285)}{\sqrt{2}} & a_{1}^{+} & K_{1}^{+} \\
a_{1}^{-} & -\frac{a_{1}^{0}}{\sqrt{2}}+\frac{f_{1}(1285)}{\sqrt{2}} & K_{1}^{0} \\
K_{1}^{-} & \bar{K}_{1}^{0} & -f_{1}(1420)
\end{array}\right)
$$


Finally we give the matrix of the baryon octet.

$$
\mathcal{B}=\left(\begin{array}{ccc}
\frac{\Sigma^{0}}{\sqrt{2}}+\frac{\Lambda^{0}}{\sqrt{6}} & \Sigma^{+} & p \\
\Sigma^{-} & -\frac{\Sigma^{0}}{\sqrt{2}}+\frac{\Lambda^{0}}{\sqrt{6}} & n \\
\Xi^{-} & \Xi^{0} & \frac{-2 \Lambda^{0}}{\sqrt{6}}
\end{array}\right)
$$

As is well known there are three types of $\mathrm{SU}(3)$ invariant couplings: the $\mathrm{F}$ and $\mathrm{D}$ types, so-called because they involve the correspondingly labeled group structure constants, and the singlet coupling, which involves the trace of the meson matrix.

$$
\begin{aligned}
\mathcal{L}_{\text {int }} & =\frac{g_{\rho N N}}{\sqrt{2}}\left[\left(1-\alpha_{V}\right) \operatorname{Tr}\left(\overline{\mathcal{B}} \gamma^{\mu}\left[\mathcal{V}_{\mu}, \mathcal{B}\right]\right)+\beta_{V} \operatorname{Tr}\left(\overline{\mathcal{B}} \gamma^{\mu} \mathcal{B}\right) \operatorname{Tr}\left(\mathcal{V}_{\mu}\right)\right. \\
& +\alpha_{V} \operatorname{Tr}\left(\overline{\mathcal{B}} \gamma^{\mu}\left\{\mathcal{V}_{\mu}, \mathcal{B}\right\}\right)+g_{A} \alpha_{A} \operatorname{Tr}\left(\overline{\mathcal{B}} \gamma^{\mu} \gamma^{5}\left\{\mathcal{A}_{\mu}, \mathcal{B}\right\}\right) \\
& \left.+g_{A}\left(1-\alpha_{A}\right) \operatorname{Tr}\left(\overline{\mathcal{B}} \gamma^{\mu} \gamma^{5}\left[\mathcal{A}_{\mu}, \mathcal{B}\right]\right)+g_{A} \beta_{A} \operatorname{Tr}\left(\overline{\mathcal{B}} \gamma^{\mu} \gamma^{5} \mathcal{B}\right) \operatorname{Tr}\left(\mathcal{A}_{\mu}\right)\right]
\end{aligned}
$$

The overall normalization of this interaction Lagrangian is determined by the coupling of the nucleons; see Eqs. (1) and (23). Four parameters are introduced: $\alpha_{V}$ and $\alpha_{A}$, which determine the relative contributions of the $\mathrm{D}$ and $\mathrm{F}$ type couplings in the vector and axialvector channels, respectively, and $\beta_{V}$ and $\beta_{A}$, which determine the corresponding singlet contributions.

There was evidence already in the 1960's that $\alpha_{A}$ was about $2 / 3$ [12]. This has been confirmed repeatedly over the years. For example, in their analysis of the spin content of the nucleon Close and Roberts [14] determined that $\alpha \approx 0.635$. As another example, Klingl, Kaiser and Weise [15] use vector meson dominance together with SU(3) symmetry to deduce $\alpha_{A}=0.68$. We shall therefore fix $\alpha_{A}=2 / 3$.

The value of $\alpha_{V}$ is determined by the requirement that the coupling of the $\phi$ vector meson, which has already been taken to be a pure $s \bar{s}$ state, to nucleons vanishes: $g_{\phi N N}=0$ [15]. This is just one aspect of the OZI rule [16]. This requirement fixes $\alpha_{V}=\left(1-\beta_{V}\right) / 2$.

We enforce the standard ratio of coupling constants of $\omega$ and $\rho$ vector mesons to nucleons, $g_{\omega N N}=3 g_{\rho N N}$, as follows from the quark model and the conventional definition of $\rho$ and $\omega$ currents, see Eqs. (44) and (45). Thus, we determine $\beta_{V}=1$. This further implies that 
$\alpha_{V}=0$. Finally, we require that the coupling of the nucleon to the $f_{1}(1420)$ meson vanishes, in analogy to the vanishing coupling of the $\phi$ meson to the nucleon. This condition fixes $\beta_{A}=\left(1-2 \alpha_{A}\right)=-1 / 3$.

The relative couplings in the vector channel with the choice $\alpha_{V}=0$ and $\beta_{V}=1$ are shown

in Table I. The absolute normalization may be inferred from the nucleon-nucleon couplings. The corresponding couplings in the axial-vector channel with the choice $\alpha_{A}=2 / 3$ and $\beta_{A}=-1 / 3$ are shown in Table II.

\section{B. Rates}

The invariant differential rates for all baryons in the octet can now be inferred. The only missing pieces are the spectral densities in the various channels. The currents as conventionally defined are, for example,

$$
\begin{aligned}
j_{\rho^{0}}^{\mu} & =\frac{1}{2}\left(\bar{u} \gamma^{\mu} u-\bar{d} \gamma^{\mu} d\right) \\
j_{\omega}^{\mu} & =\frac{1}{6}\left(\bar{u} \gamma^{\mu} u+\bar{d} \gamma^{\mu} d\right) \\
j_{\phi}^{\mu} & =-\frac{1}{3} \bar{s} \gamma^{\mu} s
\end{aligned}
$$

and so on. The spectral densities for these currents from perturbative QCD are [9]

$$
\begin{aligned}
\rho_{\rho^{0}}(s) & =\frac{s}{8 \pi^{2}}\left(1+\frac{\alpha_{s}(s)}{\pi}\right) \\
\rho_{\omega}(s) & =\frac{s}{72 \pi^{2}}\left(1+\frac{\alpha_{s}(s)}{\pi}\right) \\
\rho_{\phi}(s) & =\frac{s}{36 \pi^{2}}\left(1+\frac{\alpha_{s}(s)}{\pi}\right) .
\end{aligned}
$$

These are applicable above some threshold value which is always above the threshold for production of the corresponding baryon/anti-baryon pair, typically 1.5 to $2.5 \mathrm{GeV}^{2}$. These threshold values could be estimated rather well by making use of the QCD sum rules [9].

The rates are now determined from the SU(3) couplings, given in Tables I and II, and the spectral densities, given above. The relative weights are given in Tables III and IV from which the rates may be inferred. For convenience define the pair of functions 


$$
r_{ \pm}\left(m_{1}, m_{2}\right)=\frac{2}{(4 \pi)^{7}} \frac{F_{\mathrm{ANN}}^{2}(s)}{e^{\beta\left(E_{1}+E_{2}\right)}-1} \frac{2 s^{2}-\left(m_{1}^{2}+m_{2}^{2}\right) s-\left(m_{1}^{2}-m_{2}^{2}\right)^{2} \pm 6 m_{1} m_{2} s}{f_{\pi}^{4}}\left(1+\frac{\alpha_{s}}{\pi}\right)
$$

where the \pm corresponds to vector/axial-vector contributions. The function $F_{\mathrm{ANN}}(s)$ is a form factor, alluded to in the previous section, and determined in the following subsection. Using the symbol $r$ as shorthand notation for $E_{1} E_{2} d R / d^{3} p_{1} d^{3} p_{2}$ some examples are given below.

$$
\begin{array}{r}
r(n \bar{p})=2 r_{+}\left(m_{N}, m_{N}\right)+2 r_{-}\left(m_{N}, m_{N}\right) \\
r(p \bar{p})=2 r_{+}\left(m_{N}, m_{N}\right)+\frac{82}{81} r_{-}\left(m_{N}, m_{N}\right) \\
r(\Lambda \bar{p})=3 r_{+}\left(m_{\Lambda}, m_{N}\right)+\frac{25}{27} r_{-}\left(m_{\Lambda}, m_{N}\right) \\
r\left(\Xi^{-} \bar{\Lambda}\right)=3 r_{+}\left(m_{\Lambda}, m_{\Xi}\right)+\frac{1}{27} r_{-}\left(m_{\Lambda}, m_{\Xi}\right)
\end{array}
$$

Altogether there are 46 nonvanishing combinations of baryon/anti-baryon pairs.

The rates cannot in general be evaluated in closed form with the form factor included. However, it turns out that a very good approximation (within $10 \%$ at $T=200 \mathrm{MeV}$ ) is to evaluate $F_{\mathrm{ANN}}^{2}(s)$ at the average value $\bar{s}=\left(m_{1}+m_{2}\right)^{2}+3\left(m_{1}+m_{2}\right) T$, as discussed in the next subsection. Let us define

$$
R_{ \pm}=\int \frac{d^{3} p_{1}}{E_{1}} \frac{d^{3} p_{2}}{E_{2}} r_{ \pm}
$$

If we can evaluate $F_{\text {ANN }}^{2}$ at the average value of $s$ then the integral can be done in closed form. It is

$$
\begin{aligned}
R_{ \pm}= & \frac{9\left(1+\alpha_{s} / \pi\right) T^{8}}{4(2 \pi)^{5} f_{\pi}^{4}} z_{1}^{2} z_{2}^{2}\left\{4 z_{1} K_{1}\left(z_{1}\right) K_{2}\left(z_{2}\right)+4 z_{2} K_{1}\left(z_{2}\right) K_{2}\left(z_{1}\right)\right. \\
& \left. \pm\left(z_{1} \pm z_{2}\right)^{2} K_{1}\left(z_{1}\right) K_{1}\left(z_{2}\right)+\left[16+\left(z_{1} \pm z_{2}\right)^{2}\right] K_{2}\left(z_{1}\right) K_{2}\left(z_{2}\right)\right\} F_{\mathrm{ANN}}^{2}(\bar{s})
\end{aligned}
$$

where $z_{i}=m_{i} / T$. 


\section{Form factors}

Nucleons are composite objects, hence they have form factors that depend on the specific process. The appropriate form factor here is not the electric or magnetic form factor since we are coupling the nucleons to mesonic currents, ultimately expressed in terms of the quark fields. We can obtain a very good estimate of the relevant form factor by comparing the rate for $\bar{p} p$ production, as derived above, with the rate for $\bar{p} p$ annihilation, as obtained from kinetic theory.

The kinetic theory expression for the annihilation rate is

$$
E_{1} E_{2} \frac{d R_{\mathrm{ANN}}}{d^{3} p_{1} d^{3} p_{2}}(\bar{p} p)=\frac{4}{(2 \pi)^{6}} f\left(E_{1}\right) f\left(E_{2}\right) \frac{\sqrt{\left(p_{1} \cdot p_{2}\right)^{2}-m_{N}^{4}}}{E_{1} E_{2}} \sigma_{\mathrm{ANN}}^{\bar{p} p}(s),
$$

where $\sigma_{\mathrm{ANN}}^{\bar{p} p}(s)$ is the annihilation cross section which specifically excludes a baryon/antibaryon pair in the final state. If we approximate the thermal distributions $f(E)=$ $\exp (-E / T)$, which is a very good approximation to the Fermi-Dirac distribution at the modest temperatures of relevance here, we obtain the following simple expression.

$$
E_{1} E_{2} \frac{d R_{\mathrm{ANN}}}{d^{3} p_{1} d^{3} p_{2}}(\bar{p} p)=\frac{2}{(2 \pi)^{6}} \exp \left(-\left(E_{1}+E_{2}\right) / T\right) \sqrt{s\left(s-4 m_{N}^{2}\right)} \sigma_{\mathrm{ANN}}(s)
$$

In chemical equilibrium the rate for production must be equal to the rate for annihilation. The former is given in Eq. (52). Dropping the $f_{1}$ contribution for simplicity of presentation,

meaning that the factor $82 / 81$ is set to 1 , which is an approximation to the rate better than $0.5 \%$, we get

$$
E_{1} E_{2} \frac{d R}{d^{3} p_{1} d^{3} p_{2}}(\bar{p} p)=\frac{3}{32(2 \pi)^{7}} \exp \left(-\left(E_{1}+E_{2}\right) / T\right) \frac{s^{2}}{f_{\pi}^{4}}\left(1+\frac{\alpha_{s}}{\pi}\right) F_{\mathrm{ANN}}^{2}(s) .
$$

Equating these two yields an expression for the form factor in terms of the annihilation cross section.

$$
F_{\mathrm{ANN}}^{2}(s)=\frac{128 \pi}{3} \frac{f_{\pi}^{4}}{1+\alpha_{s} / \pi} \sqrt{1-\frac{4 m_{N}^{2}}{s}} \frac{\sigma_{\mathrm{ANN}}^{\bar{p} p}(s)}{s}
$$

The experimentally measured values of the $\bar{p} p$ annihilation cross section from several hundred $\mathrm{MeV} / \mathrm{c}$ to $8 \mathrm{GeV} / \mathrm{c}$ lab momentum has been meticulously parameterized by Cugnon and 
Vandermeulen [17]; see also the review by Dover, Gutsche, Maruyama and Faessler [18]. The fit is

$$
\sigma_{\mathrm{ANN}}^{\bar{p} p}\left(p_{L}\right)=\frac{38}{\sqrt{p_{L}}}+\frac{24}{p_{L}^{1.1}}
$$

given in mb when the lab momentum $p_{L}$ is given in $\mathrm{GeV} / \mathrm{c}$.

We have fit the form factor with the monopole function

$$
F_{\mathrm{ANN}}(s)=\frac{1}{2.21+\left(s-4 m_{N}^{2}\right) / \Lambda^{2}}
$$

where $\Lambda=1.63 \mathrm{GeV}$. This function gives a very good representation for $s-4 m_{N}^{2}>0.5$ $\mathrm{GeV}^{2}$, but overestimates $F_{\mathrm{ANN}}$ by about $10 \%$ at $s=4 m_{N}^{2}$. This overestimate is acceptable because, as we shall see below, the average value of $s-4 m_{N}^{2}$ is greater than $0.5 \mathrm{GeV}^{2}$ for $T>100 \mathrm{MeV}$.

For hyperons we choose the parameterization of the form factor to be

$$
F_{\mathrm{ANN}}(s)=\frac{1}{2.21+\left[s-\left(m_{1}+m_{2}\right)^{2}\right] / \Lambda^{2}}
$$

with the same value of $\Lambda$.

The total and elastic cross sections for $\bar{p} n$ have been measured, and the annihilation cross section estimated, for lab kinetic energies between 450 and $1068 \mathrm{MeV}$ [19] and at 3.5 GeV [20]. The annihilation cross section for $\bar{n} p$ has been explicitly measured for lab momenta between 100 and $500 \mathrm{MeV} / \mathrm{c}$ [21]. In all these cases the annihilation cross section for $\bar{p} n$ and $\bar{n} p$ has been equal to the annihilation cross section for $\bar{p} p$, albeit with large error bars in the two former cases. The difference in the thermal production rates between $\bar{p} p$ and $\bar{n} p$ calculated here is well within the error bars. [Note that the rates in Eqs. (51) and (52) do not differ much because $r_{-}\left(m_{N}, m_{N}\right)$ is rather small compared to $r_{+}\left(m_{N}, m_{N}\right)$ for $s \gtrsim 4 m_{N}^{2}$.] This is a gratifying conclusion which points to the consistency of our results compared to experimental data.

In principle the differential rates must be integrated over all energies, including the $s$ dependence of the form factor. A rough approximation is to evaluate the form factor at the 
thermal average value of $s$, which is $\bar{s}=\left(m_{1}+m_{2}\right)^{2}+3\left(m_{1}+m_{2}\right) T$, in the nonrelativistic limit and dropping terms of relative order $(T / m)^{2}$. It turns out that this approximation is good to better than $10 \%$ for temperatures less than $200 \mathrm{MeV}$.

\section{NUMERICAL RESULTS}

Consider a system at fixed temperature and volume but not necessarily in chemical equilibrium with respect to the baryons. If one of the baryons, say the anti-proton for definiteness, is out of chemical equilibrium for any reason, how long does it take for it to return to equilibrium? The rate equation for the density is

$$
\frac{d n_{\bar{p}}}{d t}=\sum_{b} R(b \bar{p})\left[1-\frac{n_{\bar{p}} n_{b}}{n_{\bar{p}}^{\text {equil }} n_{b}^{\text {equil }}}\right],
$$

where $n_{\bar{p}}^{\text {equil }}$ is the equilibrium density for anti-protons and $n_{b}^{\text {equil }}$ is the equilibrium density for the baryon species $b$. The characteristic time scale for bringing the anti-protons to equilibrium is

$$
\tau_{\bar{p}}=n_{\bar{p}}^{\text {equil }} / \sum_{b} R(b \bar{p})
$$

This characteristic time is more intuitive than the rates themselves. A fully dynamical model of the evolution of matter is required for the detailed knowledge of how the abundances develop, but for the purpose of gaining insight to the dynamics the characteristic equilibration time is perhaps more useful.

We plot this time for the proton, lambda, sigma, and cascade baryons (same as for the anti-baryons in net baryon-free matter) in Figs. 11 and 2. Figure 1 shows the times when only fluctuations in the SU(2) meson sector (no strangeness) are allowed while Fig. 2 shows the times when all mesons or currents are included (including those with strangeness). The equilibration times are strongly decreasing functions of increasing temperature. That is typical of thermal processes; thermal rates are generally strongly increasing functions of temperature because of the dominant Boltzmann factor. The time for nucleons is the 
shortest in the $\mathrm{SU}(2)$ case, which is quite natural since nucleons contain no strange quarks and they are the lightest baryon species. The lambda has the longest equilibration time as a consequence of the magnitude of its couplings to the $\mathrm{SU}(2)$ fluctuations. Going to the full SU(3) fluctuations, see Fig. 2, shortens the cascade time considerably but, surprisingly, the lambda now has the shortest equilibration time! It is interesting to note that the $S U(3)$ symmetry is broken not only by the different masses of the baryons but also by the mixing of the singlet and octet mesons.

As mentioned already, to compare with data from heavy ion collisions requires solving rate equations in an expanding and cooling system. Examples of how this may be done is described in [2,22,23]. In addition, feed down from the decay of higher mass baryon

resonances will contribute to the observed yields. But to get a rough idea, suppose that the expansion time scale is about $10 \mathrm{fm} / \mathrm{c}$ and that there are full $\mathrm{SU}(3)$ fluctuations in the system. Draw a horizontal line at $10 \mathrm{fm} / \mathrm{c}$ in Fig. 2. The intersection with the various baryon species would suggest that these baryons would reflect a freezeout temperature in the range 168 to $180 \mathrm{MeV}$, the exact value depending on the species. This is approximately the range of chemical equilibration temperatures recently seen in $\mathrm{Au}-\mathrm{Au}$ collisions at 130 and $200 \mathrm{GeV}$ at RHIC [1].

\section{CONCLUSION}

In this paper we have calculated the production of spin- $1 / 2$ baryon/anti-baryon pairs through fluctuations in the strong interaction currents. The most basic formulation used a version of the fluctuation-dissipation theorem that does not rely on the system being in thermal equilibrium. If one has a model for these fluctuations those formulas may be used directly. We evaluated them in thermal equilibrium, which gives rise to equilibration times short enough that nucleons and hyperons may very well be in chemical equilibrium in heavy ion collisions at RHIC energies.

Two natural extensions of our work arise. The first is to carry out the analogous cal- 
culation for the spin-3/2 baryon decuplet. Coupling of the strong interaction currents to spin-3/2 baryons is much more uncertain than the coupling to spin- $1 / 2$. The second is to

apply the formulas derived in this paper to a dynamical model of the expanding matter. Only then will we be able to make direct contact with RHIC experiments.

\section{ACKNOWLEDGEMENTS}

The authors thank Paul Ellis for useful discussions. This work was supported by the US Department of Energy under grant DE-FG02-87ER40328. 


\section{REFERENCES}

* On leave of absence from Bogolyubov Institute for Theoretical Physics, 252143, Kiev, Ukraine.

[1] Proceedings of Quark Matter 2002, Nantes, France, July 2002, to appear.

[2] P. Koch, B. Müller and J. Rafelski, Phys. Rep. 142, 167 (1986).

[3] R. Rapp and E. V. Shuryak, Phys. Rev. Lett. 86, 2980 (2001).

[4] T. A. DeGrand, Phys. Rev. D 30, 2001 (1984); J. Ellis, U. Heinz and H. Kowalski, Phys. Lett. B 233, 223 (1989); A. M. Srivastava, Phys. Rev. D 43, 1047 (1991); J. I. Kapusta and A. M. Srivastava, ibid. 52, 2977 (1995).

[5] D. N. Spergel, N. Turok, W. H. Press and B. S. Ryden, Phys. Rev. D 43, 1038 (1991); R. A. Leese and T. Prokopec, ibid. 44, 3749 (1991).

[6] J. I. Kapusta and S. M. H. Wong, Phys. Rev. Lett. 86, 4251 (2001).

[7] K. Rajagopal and F. Wilczek, Nucl. Phys. B 404, 577 (1993); S. Gavin and B. Muller, Phys. Lett. B 329, 486 (1994); J. I. Kapusta and A. P. Vischer, Z. Phys. C 75, 507 (1997).

[8] E. L. Feinberg, Nuovo Cim. 34A, 391 (1976); H. A. Weldon, Phys. Rev. D 28, 2007 (1983); L. D. McLerran and T. Toimela, Phys. Rev. D 31, 545 (1985); C. Gale and J. I. Kapusta, Nucl. Phys. B 357, 65 (1991).

[9] M. A. Shifman, A. I. Vainshtein and V. I. Zakharov, Nucl. Phys. B 147, 385 (1979); ibid. B 147, 448 (1979); ibid. B 147, 519 (1979); S. Narison, Z. Phys. C 14, 263 (1982); R. D. Peccei and J. Sola, Nucl. Phys. B281, 1 (1987). For finite temperature effects see: J. I. Kapusta and E. V. Shuryak, Phys. Rev. D 49, 4694 (1994).

[10] K. Kawarabayashi and M. Suzuki, Phys. Rev. Lett. 16, 255 (1966); Riazuddin and Fayazuddin, Phys. Rev. 147, 1071 (1966).

[11] Particle Data Group, D. E. Groom et al., Eur. Phys. J. C 15, 1 (2000). 
[12] S. Gasiorowicz, Elementary Particle Physics, Wiley \& Sons, 1966.

[13] T.-P. Cheng and L.-F. Li, Gauge Theory of Elementary Particle Physics, Oxford University Press, 1984.

[14] F. E. Close and R. G. Roberts, Phys. Lett. B 316, 165 (1993).

[15] F. Klingl, N. Kaiser and W. Weise, Z. Phys. A 356, 193 (1996); Nucl. Phys. A 624, 527 (1997).

[16] S. Okubo, Phys. Lett. 5, 165 (1963); G. Zweig, Preprint CERN-TH-412 (1964) (unpublished); J. Iizuka, Prog. Theor. Phys. Suppl. 37, 21 (1966).

[17] J. Cugnon and J. Vandermeulen, Annales de Physique (France), 14, 49 (1989).

[18] C. Dover, T. Gutsche, M. Maruyama and A. Faessler, Prog. Part. Nucl. Phys. 29, 87 (1992).

[19] T. Elioff, L. Agnew, O. Chamberlain and H. M. Steiner, Phys. Rev. 128, 869 (1962).

[20] B. G. Reynolds, K. E. Weaver, J. M. Bishop, D. O. Huwe and J. A. Malko, Phys. Rev. D 2, 1767 (1970).

[21] T. Armstrong et al., Phys. Rev. D 36, 659 (1987).

[22] J. Kapusta and A. Mekjian, Phys. Rev. D 33, 1304 (1986).

[23] C. Greiner, preprint nucl-th/0208080 (unpublished). 
Table I: Relative strength of vector couplings for $\alpha_{V}=0$ and $\beta_{V}=1$. The names of vector mesons that couple to the given baryon/anti-baryon pairs are shown explicitly.

\begin{tabular}{|c|c|c|c|c|c|c|c|c|}
\hline & $p$ & $n$ & $\Lambda$ & $\Sigma^{0}$ & $\Sigma^{+}$ & $\Sigma^{-}$ & $\Xi^{0}$ & $\Xi^{-}$ \\
\hline $\bar{p}$ & $\begin{array}{l}\rho^{0} \\
3 \omega\end{array}$ & $\sqrt{2} \rho^{+}$ & $-\sqrt{3} K_{*}^{+}$ & $-K_{*}^{+}$ & $-\sqrt{2} K_{*}^{0}$ & 0 & 0 & 0 \\
\hline $\bar{n}$ & $\sqrt{2} \rho^{-}$ & $\begin{array}{l}-\rho^{0} \\
3 \omega\end{array}$ & $-\sqrt{3} K_{*}^{0}$ & $K_{*}^{0}$ & 0 & $-\sqrt{2} K_{*}^{+}$ & 0 & 0 \\
\hline $\bar{\Lambda}$ & $-\sqrt{3} K_{*}^{-}$ & $-\sqrt{3} \bar{K}_{*}^{0}$ & $\begin{array}{c}2 \omega \\
-\sqrt{2} \phi\end{array}$ & 0 & 0 & 0 & $\sqrt{3} K_{*}^{0}$ & $\sqrt{3} K_{*}^{+}$ \\
\hline $\bar{\Sigma}^{0}$ & $-K_{*}^{-}$ & $\bar{K}_{*}^{0}$ & 0 & $\begin{array}{c}2 \omega \\
-\sqrt{2} \phi\end{array}$ & $-2 \rho^{-}$ & $2 \rho^{+}$ & $-K_{*}^{0}$ & $K_{*}^{+}$ \\
\hline $\bar{\Sigma}^{+}$ & $-\sqrt{2 \bar{K}_{*}^{0}}$ & 0 & 0 & $-2 \rho^{+}$ & $\begin{array}{c}2 \rho^{0} \\
2 \omega \\
-\sqrt{2} \phi\end{array}$ & 0 & $\sqrt{2} K_{*}^{+}$ & 0 \\
\hline $\bar{\Sigma}^{-}$ & 0 & $-\sqrt{2} K_{*}^{-}$ & 0 & $2 \rho^{-}$ & 0 & $\begin{array}{c}2 \rho^{0} \\
2 \omega \\
-\sqrt{2} \phi\end{array}$ & 0 & $-\sqrt{2} K_{*}^{0}$ \\
\hline $\bar{\Xi}^{0}$ & 0 & 0 & $\sqrt{3 \bar{K}_{*}^{0}}$ & $-\bar{K}_{*}^{0}$ & $\sqrt{2} K_{*}^{-}$ & 0 & $\begin{array}{c}\rho^{0} \\
\omega \\
-2 \sqrt{2} \phi\end{array}$ & $-\sqrt{2} \rho^{+}$ \\
\hline $\bar{\Xi}^{-}$ & 0 & 0 & $\sqrt{3} K_{*}^{-}$ & $K_{*}^{-}$ & 0 & $\sqrt{2} \bar{K}_{*}^{0}$ & $-\sqrt{2} \rho^{-}$ & $\begin{array}{c}-\rho^{0} \\
\omega \\
-2 \sqrt{2} \phi\end{array}$ \\
\hline
\end{tabular}


Table II: Relative strength of axial-vector couplings for $\alpha_{A}=2 / 3$ and $\beta_{A}=-1 / 3$. The names of axial-vector mesons that couple to the given baryon/anti-baryon pairs are shown explicitly. The notation $\tilde{f}_{1}$ stands for $f_{1}(1420)$.

\begin{tabular}{|c|c|c|c|c|c|c|c|c|}
\hline & $p$ & $n$ & $\Lambda$ & $\Sigma^{0}$ & $\Sigma^{+}$ & $\Sigma^{-}$ & $\Xi^{0}$ & $\Xi^{-}$ \\
\hline $\bar{p}$ & $\begin{array}{c}a_{1}^{0} \\
\frac{1}{3} f_{1}\end{array}$ & $\sqrt{2} a_{1}^{+}$ & $-\frac{5}{3 \sqrt{3}} K_{1}^{+}$ & $\frac{1}{3} K_{1}^{+}$ & $\frac{\sqrt{2}}{3} K_{1}^{0}$ & 0 & 0 & 0 \\
\hline $\bar{n}$ & $\sqrt{2} a_{1}^{-}$ & $\begin{array}{l}-a_{1}^{0} \\
\frac{1}{3} f_{1}\end{array}$ & $-\frac{5}{3 \sqrt{3}} K_{1}^{0}$ & $-\frac{1}{3} K_{1}^{0}$ & 0 & $\frac{\sqrt{2}}{3} K_{1}^{+}$ & 0 & 0 \\
\hline $\bar{\Lambda}$ & $-\frac{5}{3 \sqrt{3}} K_{1}^{-}$ & $-\frac{5}{3 \sqrt{3}} \bar{K}_{1}^{0}$ & $\begin{array}{c}-\frac{2}{9} f_{1} \\
-\frac{5 \sqrt{2}}{9} \tilde{f}_{1}\end{array}$ & $\frac{4}{3 \sqrt{3}} a_{1}^{0}$ & $\frac{4}{3 \sqrt{3}} a_{1}^{-}$ & $\frac{4}{3 \sqrt{3}} a_{1}^{+}$ & $\frac{1}{3 \sqrt{3}} K_{1}^{0}$ & $\frac{1}{3 \sqrt{3}} K_{1}^{+}$ \\
\hline $\bar{\Sigma}^{0}$ & $\frac{1}{3} K_{1}^{-}$ & $-\frac{1}{3} \bar{K}_{1}^{0}$ & $\frac{4}{3 \sqrt{3}} a_{1}^{0}$ & $\begin{array}{c}\frac{2}{3} f_{1} \\
-\frac{\sqrt{2}}{3} \tilde{f}_{1}\end{array}$ & $-\frac{2}{3} a_{1}^{-}$ & $\frac{2}{3} a_{1}^{+}$ & $-K_{1}^{0}$ & $K_{1}^{+}$ \\
\hline $\bar{\Sigma}^{+}$ & $\frac{\sqrt{2}}{3} \bar{K}_{1}^{0}$ & 0 & $\frac{4}{3 \sqrt{3}} a_{1}^{+}$ & $-\frac{2}{3} a_{1}^{+}$ & $\begin{array}{l}\frac{2}{3} a_{1}^{0} \\
\frac{2}{3} f_{1} \\
\frac{\sqrt{2}}{3} \tilde{f}_{1}\end{array}$ & 0 & $\sqrt{2} K_{1}^{+}$ & 0 \\
\hline $\bar{\Sigma}^{-}$ & 0 & $\frac{\sqrt{2}}{3} K_{1}^{-}$ & $\frac{4}{3 \sqrt{3}} a_{1}^{-}$ & $\frac{2}{3} a_{1}^{-}$ & 0 & $\begin{array}{c}-\frac{2}{3} a_{1}^{0} \\
\frac{2}{3} f_{1} \\
\frac{\sqrt{2}}{3} \tilde{f}_{1}\end{array}$ & 0 & $\sqrt{2} K_{1}^{0}$ \\
\hline $\bar{\Xi}^{0}$ & 0 & 0 & $\frac{1}{3 \sqrt{3}} \bar{K}_{1}^{0}$ & $-\bar{K}_{1}^{0}$ & $\sqrt{2} K_{1}^{-}$ & 0 & $\begin{array}{c}-\frac{1}{3} a_{1}^{0} \\
-\frac{1}{3} f_{1} \\
-\frac{2 \sqrt{2}}{3} \tilde{f}_{1}\end{array}$ & $\frac{\sqrt{2}}{3} a_{1}^{+}$ \\
\hline $\bar{\Xi}^{-}$ & 0 & 0 & $\frac{1}{3 \sqrt{3}} K_{1}^{-}$ & $K_{1}^{-}$ & 0 & $\sqrt{2} \bar{K}_{1}^{0}$ & $\frac{\sqrt{2}}{3} a_{1}^{-}$ & $\begin{array}{c}\frac{1}{3} a_{1}^{0} \\
-\frac{1}{3} f_{1} \\
-\frac{2 \sqrt{2}}{3} \tilde{f}_{1}\end{array}$ \\
\hline
\end{tabular}


Table III: Numerical vector channel multipliers in the expressions for the rates $\left(\alpha_{V}=0\right.$ and $\left.\beta_{V}=1\right)$.

\begin{tabular}{|c||c|c|c|c|c|c|c|c|}
\hline & $p$ & $n$ & $\Lambda$ & $\Sigma^{0}$ & $\Sigma^{+}$ & $\Sigma^{-}$ & $\Xi^{0}$ & $\Xi^{-}$ \\
\hline \hline $\bar{p}$ & 2 & 2 & 3 & 1 & 2 & 0 & 0 & 0 \\
\hline $\bar{n}$ & 2 & 2 & 3 & 1 & 0 & 2 & 0 & 0 \\
\hline $\bar{\Lambda}$ & 3 & 3 & $\frac{8}{9}$ & 0 & 0 & 0 & 3 & 3 \\
\hline $\bar{\Sigma}^{0}$ & 1 & 1 & 0 & $\frac{8}{9}$ & 4 & 4 & 1 & 1 \\
\hline $\bar{\Sigma}^{+}$ & 2 & 0 & 0 & 4 & $\frac{44}{9}$ & 0 & 2 & 0 \\
\hline $\bar{\Sigma}^{-}$ & 0 & 2 & 0 & 4 & 0 & $\frac{44}{9}$ & 0 & 2 \\
\hline $\bar{\Xi}^{0}$ & 0 & 0 & 3 & 1 & 2 & 0 & $\frac{26}{9}$ & 2 \\
\hline $\bar{\Xi}^{-}$ & 0 & 0 & 3 & 1 & 0 & 2 & 2 & $\frac{26}{9}$ \\
\hline
\end{tabular}

Table IV: Numerical axial-vector channel multipliers in the expressions for the rates $\left(\alpha_{A}=\right.$ $2 / 3$ and $\left.\beta_{A}=-1 / 3\right)$.

\begin{tabular}{|c||c|c|c|c|c|c|c|c|}
\hline & $p$ & $n$ & $\Lambda$ & $\Sigma^{0}$ & $\Sigma^{+}$ & $\Sigma^{-}$ & $\Xi^{0}$ & $\Xi^{-}$ \\
\hline \hline $\bar{p}$ & $\frac{82}{81}$ & 2 & $\frac{25}{27}$ & $\frac{1}{9}$ & $\frac{2}{9}$ & 0 & 0 & 0 \\
\hline $\bar{n}$ & 2 & $\frac{82}{81}$ & $\frac{25}{27}$ & $\frac{1}{9}$ & 0 & $\frac{2}{9}$ & 0 & 0 \\
\hline $\bar{\Lambda}$ & $\frac{25}{27}$ & $\frac{25}{27}$ & $\frac{104}{729}$ & $\frac{16}{27}$ & $\frac{16}{27}$ & $\frac{16}{27}$ & $\frac{1}{27}$ & $\frac{1}{27}$ \\
\hline $\bar{\Sigma}^{0}$ & $\frac{1}{9}$ & $\frac{1}{9}$ & $\frac{16}{27}$ & $\frac{8}{81}$ & $\frac{4}{9}$ & $\frac{4}{9}$ & 1 & 1 \\
\hline $\bar{\Sigma}^{+}$ & $\frac{2}{9}$ & 0 & $\frac{16}{27}$ & $\frac{4}{9}$ & $\frac{44}{81}$ & 0 & 2 & 0 \\
\hline $\bar{\Sigma}^{-}$ & 0 & $\frac{2}{9}$ & $\frac{16}{27}$ & $\frac{4}{9}$ & 0 & $\frac{44}{81}$ & 0 & 2 \\
\hline $\bar{\Xi}^{0}$ & 0 & 0 & $\frac{1}{27}$ & 1 & 2 & 0 & $\frac{26}{81}$ & $\frac{2}{9}$ \\
\hline $\bar{\Xi}^{-}$ & 0 & 0 & $\frac{1}{27}$ & 1 & 0 & 2 & $\frac{2}{9}$ & $\frac{26}{81}$ \\
\hline
\end{tabular}




\section{FIGURES}

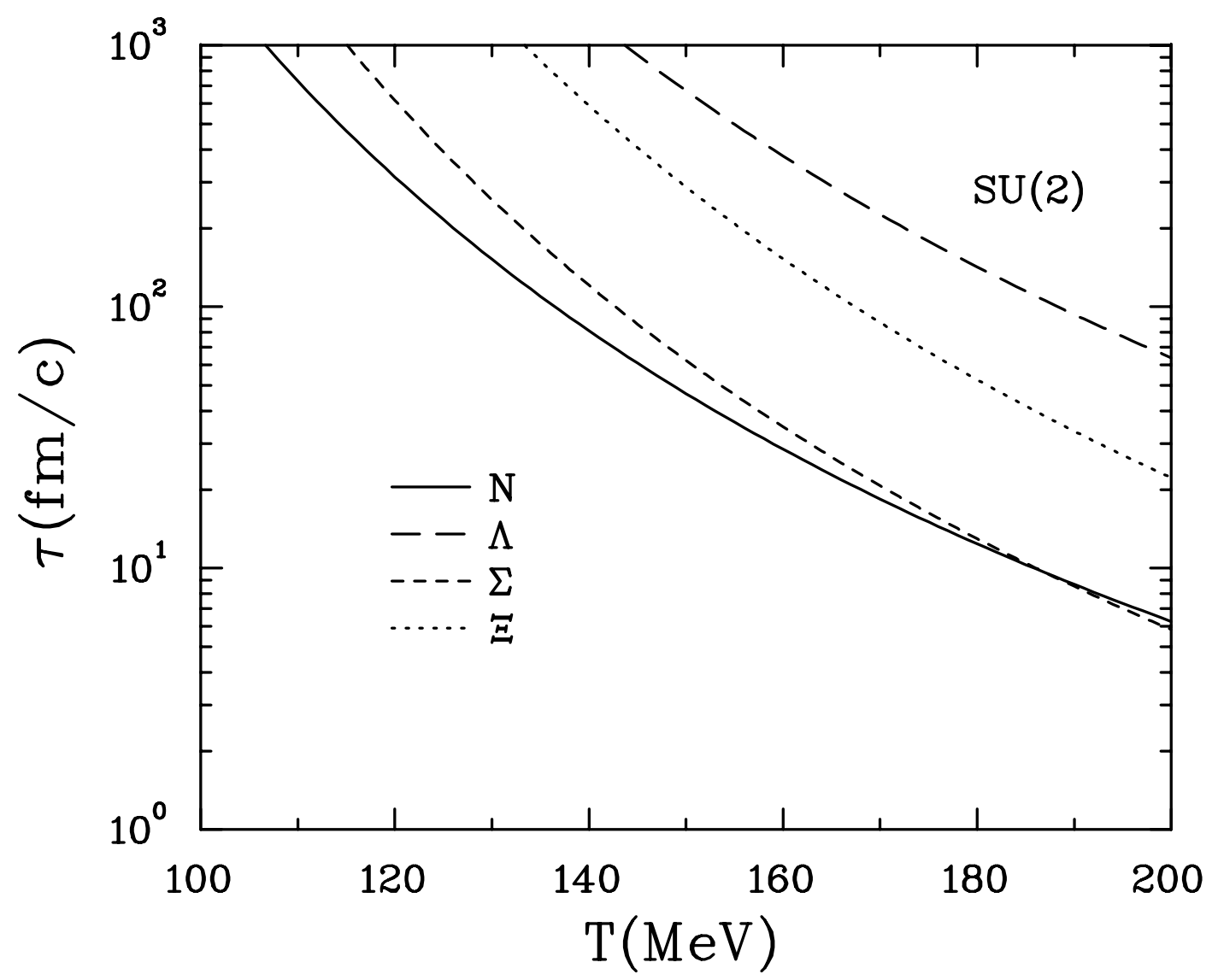

FIG. 1. Numerical results for equilibration times in the case when only the fluctuations in the $S U(2)$ meson sector are taken into accout. 


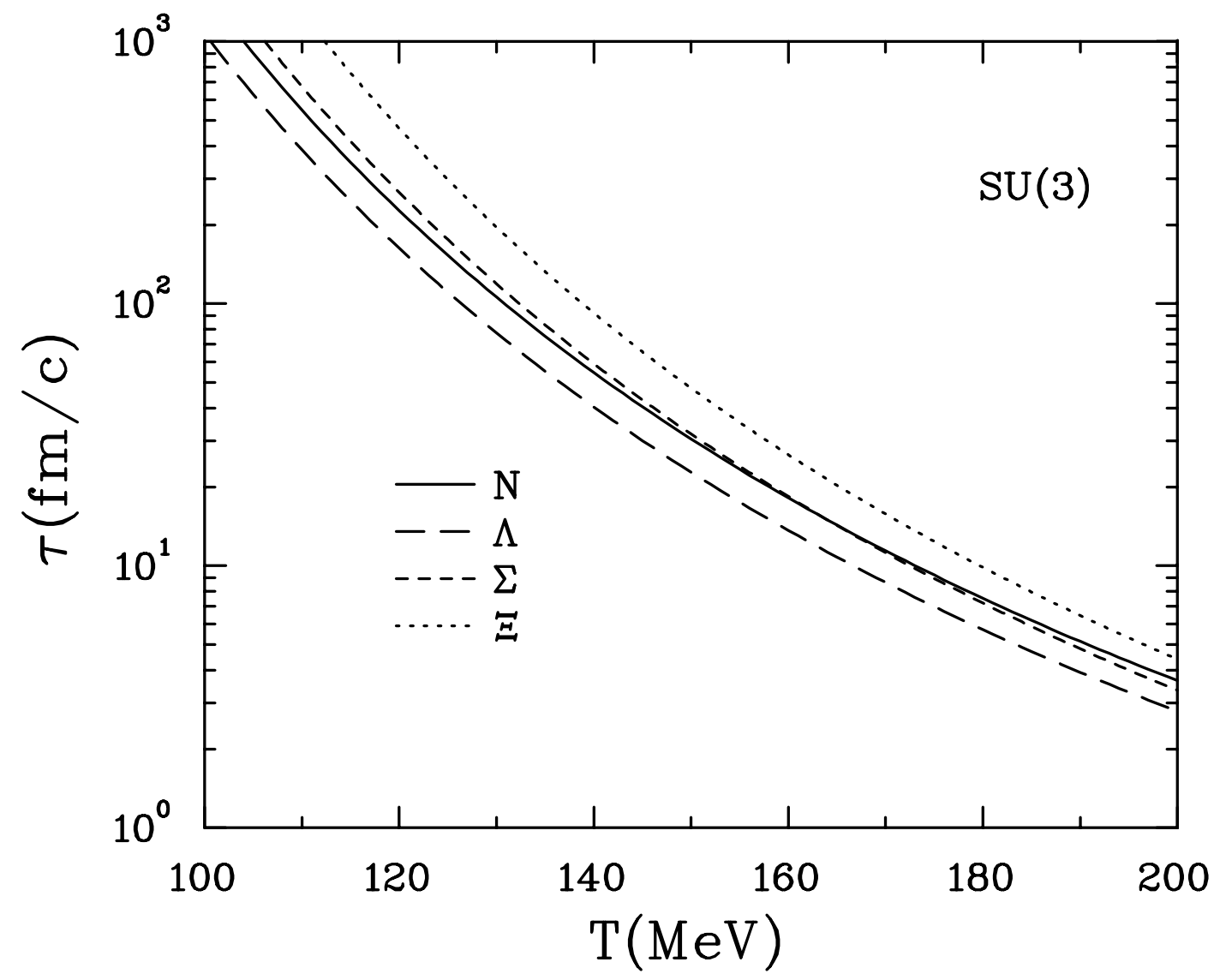

FIG. 2. Numerical results for equilibration times in the case when all fluctuations in the $S U(3)$ meson sector are taken into accout. 